\title{
Spatial mobility fluctuation induced giant linear magnetoresistance in multilayered graphene foam
}

\author{
Peng Li, ${ }^{1}$ Qiang Zhang, ${ }^{1}$ Xin He, ${ }^{1}$ Wencai Ren, ${ }^{2}$ Hui-Ming Cheng, ${ }^{2}$ and Xi-xiang Zhang ${ }^{1, *}$ \\ ${ }^{1}$ Division of Physical Science and Engineering, King Abdullah University of Science and Technology (KAUST), Thuwal 23955, \\ Kingdom of Saudi Arabia \\ ${ }^{2}$ Shenyang National Laboratory for Materials Science, Institute of Metal Research, Chinese Academy of Sciences, \\ Shenyang 110016, China \\ (Received 16 January 2016; revised manuscript received 24 May 2016; published 5 July 2016)
}

\begin{abstract}
Giant, positive, and near-temperature-independent linear magnetoresistance (LMR), as large as 340\%, was observed in graphene foam with a three-dimensional flexible network. Careful analysis of the magnetoresistance revealed that Shubnikov-de Haas $(\mathrm{SdH})$ oscillations occurred at low temperatures and decayed with increasing temperature. The average classical mobility ranged from $300(2 \mathrm{~K})$ to $150(300 \mathrm{~K}) \mathrm{cm}^{2} \mathrm{~V}^{-1} \mathrm{~s}^{-1}$, which is much smaller than that required by the observed SdH oscillations. To understand the mechanism behind the observation, we performed the same measurements on the microsized graphene sheets that constitute the graphene foam. Much more pronounced SdH oscillations superimposed on the LMR background were observed in these microscaled samples, which correspond to a quantum mobility as high as $26,500 \mathrm{~cm}^{2} \mathrm{~V}^{-1} \mathrm{~s}^{-1}$. Moreover, the spatial mobility fluctuated significantly from $64,200 \mathrm{~cm}^{2} \mathrm{~V}^{-1} \mathrm{~s}^{-1}$ to $1370 \mathrm{~cm}^{2} \mathrm{~V}^{-1} \mathrm{~s}^{-1}$, accompanied by a variation of magnetoresistance from near $20,000 \%$ to less than $20 \%$. The presence of SdH oscillations actually excludes the possibility that the observed LMR originated from the extreme quantum limit, because this would demand all electrons to be in the first Landau level. Instead, we ascribe the large LMR to the second case of the classical Parish and Littlewood model, in which spatial mobility fluctuation dominates electrical transport. This is an experimental confirmation of the Parish and Littlewood model by measuring the local mobility randomly (by measuring the microsized graphene sheets) and finding the spatial mobility fluctuation.
\end{abstract}

DOI: 10.1103/PhysRevB.94.045402

\section{INTRODUCTION}

Generally, the magnetoresistance (MR) of a conductor is negligibly small, follows a quadratic dependence on the applied magnetic field, and saturates at $B>\mu^{-1}$, where $B$ and $\mu$ are the magnetic field and the carrier mobility, respectively. The size of MR in metals is usually less than a few percent. Linear magnetoresistance (LMR) is a phenomenon that has been observed in several materials: zero-gap semiconductors $\mathrm{Ag}_{2 \pm \delta} \mathrm{Se}$ [1], $\mathrm{Ag}_{2 \pm \delta} \mathrm{Te}$ [2], and Sb-rich InSb [3], topological insulators $\mathrm{Bi}_{2} \mathrm{Te}_{3}[4,5]$, and recently Dirac and Weyl semimetals TlBiSSe, $\mathrm{WTe}_{2}$, and $\mathrm{Cd}_{3} \mathrm{As}_{2}$ [6-9], as well as epitaxial graphene films $[10,11]$. In epitaxial, multilayered graphene samples, near-linear and positive MR has been observed, ranging from negligibly small up to $200 \%[10,11]$. Interestingly, such a large MR was also observed in a multilayer graphene film with current flowing perpendicular to the film plane [12]. Owing to the characteristics of the observed LMR (e.g., linear field dependence, temperature indepenece), LMR has attracted considerable attention for both fundamental interests and potential applications, such as in Megagauss sensors and other magnetic devices [13-17]. Therefore, a deeper understanding of the physics behind the LMR is of great importance.

To date, two models have been proposed to explain LMR: the classical model (Parish and Littlewood, PL) [18,19] and the quantum model (Abrikosov) [20,21]. Within the classical model, it is argued that the magnetotransport properties of the material and the LMR are governed by spatial mobility fluctuations rather than the mobility itself $[3,19,22,23]$. Two

\footnotetext{
*Author to whom all correspondence should be addressed: xixiang.zhang@kaust.edu.sa
}

cases were studied numerically based on the properties of the mobility, and LMR was observed in both cases. In the first case, the average mobility is larger than the mobility fluctuation $(\Delta \mu /\langle\mu\rangle<1)$, whereas in the second case, the mobility fluctuation is larger than the average mobility $(\Delta \mu /\langle\mu\rangle>1)$. Experimentally, it is very difficult to obtain the spatial mobility fluctuation, because it is extremely challenging to directly measure the local mobility in a random location in a sample. One possibility is to estimate the local mobility based on the sample properties; however, this requires detailed information about the local crystalline structure, composition, and defects, etc., in an inhomogeneous sample [24]. Meanwhile, in the quantum model, LMR appears if the condition "extreme quantum limit, $E_{F}<\hbar \omega_{c}$ " (where $E_{F}$ and $\hbar \omega_{c}$ are the Fermi energy and Landau levels separation, respectively) is fulfilled, which is typically observed in materials with a zero energy gap and Dirac linear dispersion [4].

Indeed, a conclusive explanation for the observed giant and positive LMR has not been found. For example, even when both models have been applied to the same material system, to interpret $\mathrm{LMR}$ in the topological insulator $\mathrm{Bi}_{2} \mathrm{Te}_{3}[4,5]$, the LMR in nanosheets of $\mathrm{Bi}_{2} \mathrm{Te}_{3}$ was ascribed to the quantum MR model, whereas in another work, the LMR observed in $\mathrm{Bi}_{2} \mathrm{Te}_{3}$ films was interpreted within the classical PL model, in which mobility fluctuations dominated the electrical transport [5]. The temperature-independent LMR observed in epitaxial, multilayered graphene samples is generally attributed to the quantum model $[10,11]$ because of its well-known linear energy spectrum. This Dirac core energy structure gives a near-zero effective mass, easily satisfying the extreme quantum limit. However, the prerequisites for Shubnikovde Haas $(\mathrm{SdH})$ oscillations and quantum LMR appear to be contradictory. $E_{F}>\hbar \omega_{c}$ is required for observing $\mathrm{SdH}$ 
oscillations, whereas $E_{F}<\hbar \omega_{c}$ is demanded to satisfy the extreme quantum conditions given by Abrikosov, in which all electrons fall into the first Landau level. The origin of LMR in graphene films $[10,11,25,26]$ and even Dirac and Weyl semimetals [7-9] is therefore inconclusive. Therefore, a better understanding of the positive, nonsaturating, and near-linear MR in various systems is essential for not only the fundamental science, but also for potential applications.

In this work, a giant LMR (340\%, @9 T) and clear $\mathrm{SdH}$ oscillations were found in flexible, inhomogeneous, macroscopic three-dimensional (3D) polycrystalline graphene foams. Several microsized graphene sheets that constituted the 3D graphene foam were studied to gain a deeper understanding of the large sample. The results on the microsamples clearly demonstrated the existence of strong mobility fluctuation. This experiment shows that local mobility can be measured at random locations. In this work, the observed $\mathrm{SdH}$ oscillations are attributed to the high-quality graphene sheets ranging in lateral size from 8 to $10 \mu \mathrm{m}$ with high mobility, whereas the giant LMR has been interpreted within the spatial mobility fluctuation in an imhomogeneous conducting graphene foam network, serving as a very strong experimental confirmation of the PL model.

\section{EXPERIMENTAL DETAILS}

Inhomogeneous, polycrystalline graphene foams were fabricated by template-directed chemical vapor deposition (CVD) using a nickel skeleton support, a technique which has previously been reported [27]. Raman experiments were performed on a Hariba LabRAM HR spectrometer, and the morphology and microstructure of samples were characterized by scanning electron microscopy (SEM; Nova Nano 630, FEI). The layered structure of the graphene foam was examined using high-resolution transmission electron microscopy (HRTEM; Monochromated Cs Image FEI, $60 \mathrm{kV}$ ). Since the Ni skeleton was completely etched off, no Ni residuals or other impurities were detected in the graphene foams by energy-dispersive $\mathrm{X}$-ray spectroscopy (EDX) installed in both SEM and TEM. The magnetic measurement on the graphene foam using a Quantum Design SQUID magnetometer showed no detectable ferromagnetic signal coming from the Ni residual, confirming the EDX results. Magnetotransport properties, including MR and anisotropic MR, were measured with a Quantum Design Physical Property Measurement System (PPMS) equipped with a sample rotator, which can alter the angle between the magnetic field and the current. The temperature during measurements ranged from $2 \mathrm{~K}$ to $300 \mathrm{~K}$, and the current applied while measuring transport was $10 \mu \mathrm{A}$. The MR in this work is defined as $\mathrm{MR}=\left(R_{B}-R_{0}\right) / R_{0}$, where $R_{0}$ and $R_{\mathrm{B}}$ are the resistance at zero and $B$ magnetic fields, respectively. Microsize graphene sheets were obtained by breaking the foam in an ultrasonic bath, and then the resulting sheets were transferred onto $\mathrm{Si} / \mathrm{SiO}_{2}(280 \mathrm{~nm})$ wafers. The Ti $(10 \mathrm{~nm}) / \mathrm{Au}$ $(100 \mathrm{~nm})$ electrodes were fabricated on the high-quality samples (good crystallinity, flat, large and regularly shaped sheets) using standard e-beam lithography (EBL) and e-beam evaporation. The detailed nanofabrication process is provided in Supplemental Material Fig. S1 [28].

\section{RESULTS AND DISCUSSION}

The low-magnification SEM image in Fig. 1(a) shows the interconnected skeleton of graphene foam, indicating the disordered, 3D conduction path. Figure 1(b) shows the high-magnification SEM image of a graphene foam scaffold. The thick, bright lines that form the network pattern replicated the grain boundaries of the Ni skeleton, which may also define the size of graphene grains. The thin, bright lines within the graphene grains represent the natural ripples and wrinkles caused by the thermal expansion coefficient difference between nickel and graphene [29]. The inset of Fig. 1(a) shows the typical Raman spectrum of graphene foam, collected at a random site, with its asymmetrical two-dimensional (2D) peak located at $2720 \mathrm{~cm}^{-1}$, indicating that the graphene foam grown with CVD formed a multilayered structure. Peak D at $1350 \mathrm{~cm}^{-1}$ indicates the existence of imperfections in the graphene. The HRTEM images shown in the upper (planar) and lower (cross sectional) insets of Fig. 1(b) indicate that the foam consists of multiple layers of graphene separated by $0.34 \mathrm{~nm}$ interplanar spaces.

Figure 1(c) represents the typical magnetic field dependence of the MR in multilayered polycrystalline graphene foam from $2 \mathrm{~K}$ to $300 \mathrm{~K}$. Several features in these MR curves are distinguishable; for example, the MR is (i) positive; (ii) relatively large (as high as $340 \%$ at $9 \mathrm{~T}$ and room temperature); and (iii) unsaturated at high fields with near-linear proportions to the magnetic field over the entire temperature range. This positive LMR observed in the multilayered graphene foam was much higher than previously reported values in single-layered graphene (negligible small) [30] or multilayered epitaxial graphene $(180 \%$ at $9 \mathrm{~T}[10] ; 50 \%$ at $9 \mathrm{~T}[11] ; 100 \%$ at $14 \mathrm{~T}$ [12]). We also measured the Hall resistance of graphene foam at different temperatures $(2-300 \mathrm{~K})$ to obtain more information about the transport properties of the material, as shown in the inset of Fig. 1(c). A linear dependence of Hall resistance on the applied magnetic field with positive slope is observed over the whole temperature range. The positive slope indicates that a hole-like carrier dominated the electrical transport. The fact that the slope increases with decreasing temperature suggests that the carrier density decreases as the material is cooled down. Using these experimental data and longitudinal resistance data, we obtained the average classical carrier mobility based on $\mu=R_{H} / \rho_{x x}$. The average mobility data will be discussed later.

To gain a deeper understanding of the bahavior and the underlying mechanisms of the giant LMR [Fig. 1(c)], we calculated the first-order derivative of the curves with respect to magnetic field following the same procedure used previously for MR data obtained from topological insulators [31,32]. The obtained derivatives were then plotted as a function of applied magnetic fields in Fig. 1(d). Interestingly, the low-temperture data clearly show peaks and valleys at certain magnetic fields [also see the amplification in the inset of Fig. 1(d)], and the oscillation amplitudes rapidly decrease and finally vanish as temperature increases, strongly indicating the presence of $\mathrm{SdH}$ oscillations in the macroscopical graphene foam [31,33,34].

To understand the dimensionality of the electrical transport in this graphene foam, we performed anisotropic MR measurements at different magnetic field directions with 

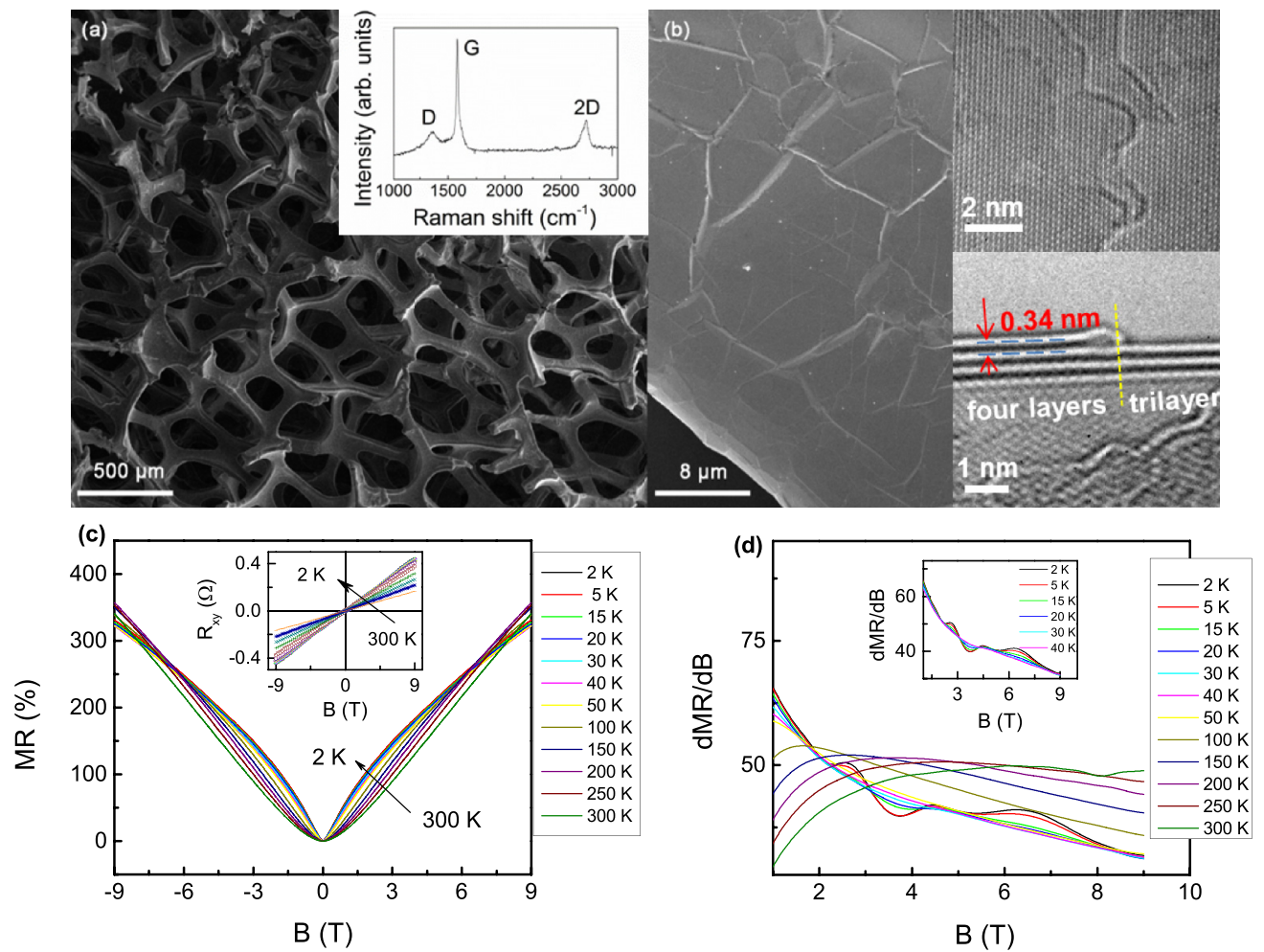

FIG. 1. (a) Low-resolution SEM image of graphene foam with a 3D network. The inset shows a typical Raman spectrum of graphene foam. (b) High-resolution SEM image of a graphene foam scaffold; upper and lower insets are planer and cross-sectional HRTEM, respectively, of a multilayered graphene sheet. (c) Field dependence of the MR of macroscopic graphene foam at various temperatures. Inset shows the Hall effect data measured on the same sample. (d) Derivative of MR with respect to magnetic field, $d \mathrm{MR} / d B$, at different temperatures. Inset clearly shows the oscillations in the low-temperature curves of $d \mathrm{MR} / d B$ versus $B$.

respect to the current (see Supplemental Material Fig. S2 and Note 1 [35]). Based on the angular dependence of MR and their first-order derivatives with respect to the magnetic field, we can conclude that the electrical transport in the graphene foam was dominated by $2 \mathrm{D}$ transport with some 3D component. This can be understood as follows. The 2D transport comes from the 2D Fermi surface of multilayer graphene sheets. The 3D component should be due to the 3D conducting network formed by the $2 \mathrm{D}$ graphene sheets. We also exclude the effect of residuals of the magnetic nickel skeleton and magnetic impurity on the MR of graphene foams (see Supplemental Material Fig. S3 and Note 2 [36]).

Now, we attempt to interpret the giant LMR within the quantum MR model [20,21], which demands the separation of Landau levels being larger than the Fermi energy of the material, i.e., $\hbar \omega_{c}>E_{F}$. In this case, only the first Landau levels are occupied by carriers. However, $\mathrm{SdH}$ oscillations originate from the successive emptying of Landau levels with an increasing magnetic field [Fig. 1(d)], indicating that quantum MR is unlikely to be the origin of the large LMR in graphene foam.

To support this argument and reveal more about the transport mechanism, we fabricated several microsized samples using the standard EBL technique and the graphene sheets separated from the large graphene foam. Figure 2(a) shows the MR (electrodes 1 and 2) curves measured on one of the typical samples at low temperatures, and the inset of Fig. 2(a) shows the optical image of the sample with micropatterned electrical contacts. Much more pronounced SdH oscillations are superimposed onto the LMR background for low-temperature data.
Subtracting the linear background yields more distinct $\mathrm{SdH}$ oscillations in Fig. 2(b). As shown in Fig. 2(c), linear fitting of the Landau index number $(n)$ as a function of $1 / B$ yields the $\mathrm{SdH}$ oscillation frequency, $B_{F}$, and Berry phase, being extracted from the slope and from the intercept, respectively. Given the 2D Fermi surface of our sample (Fig. S2), the slope in Fig. 2(c) corresponds to an $\mathrm{SdH}$ oscillation frequency of $B_{F} \sim 4.66 \mathrm{~T}$. The Fermi surface radius, $k_{F}=\sqrt{2 e B_{F} / \hbar}=1.37 \times 10^{8} \mathrm{~m}^{-1}$, and carrier density, $n_{S}=g_{s} g_{v} k_{F}^{2} / 4 \pi=k_{F}^{2} / \pi=4.49 \times$ $10^{11} / \mathrm{cm}^{2}$, were then deduced using the extracted value of $B_{F}$, in which $g_{s}$ and $g_{v}$ are, respectively, the degeneracy of spin and valley. To analyze the $\mathrm{SdH}$ oscillations more qualitatively, we fitted the entire oscillatory component (or curve) with the standard Lifshitz-Kosevich (LK) theory for a 2D system [37,38]:

$$
\frac{\Delta \rho}{\rho} \propto \frac{\lambda}{\sinh \lambda} e^{-\lambda_{D}} \cos 2 \pi\left[\frac{B_{F}}{B}+\frac{1}{2}+\beta\right],
$$

where $\lambda=2 \pi^{2} k_{\mathrm{B}} T m^{*} / \hbar e \mathrm{~B}=2 \pi^{2} k_{\mathrm{B}} T / \Delta E_{N}(B) \quad$ [Landau level difference $\left.\Delta E_{N}(B)=\frac{\hbar e B}{m^{*}}\right]$ and $\lambda_{D}=2 \pi^{2} k_{\mathrm{B}} T_{D} m^{*} /$ $\hbar e B=2 \pi^{2} k_{\mathrm{B}} T_{D} / \Delta E_{N}(B), m^{*}$ is the cyclotron effective mass of the carriers, and $T_{D}=h / 4 \pi^{2} \tau k_{\mathrm{B}}$ is the Dingle temperature, which represents the broadening of the Landau level. Here, $\tau$ is the quantum lifetime of the carriers due to scattering. Figure 2(d) shows the temperature dependence of $\mathrm{SdH}$ oscillation amplitude at different magnetic fields, where the solid lines are the best fits to Eq. (1). The linear fitting of the Landau level difference with the magnetic field, $\Delta E_{N}(B)$, leads to the cyclotron effective mass, $m^{*}=0.042 m_{0}$ from Fig. 2(e). 

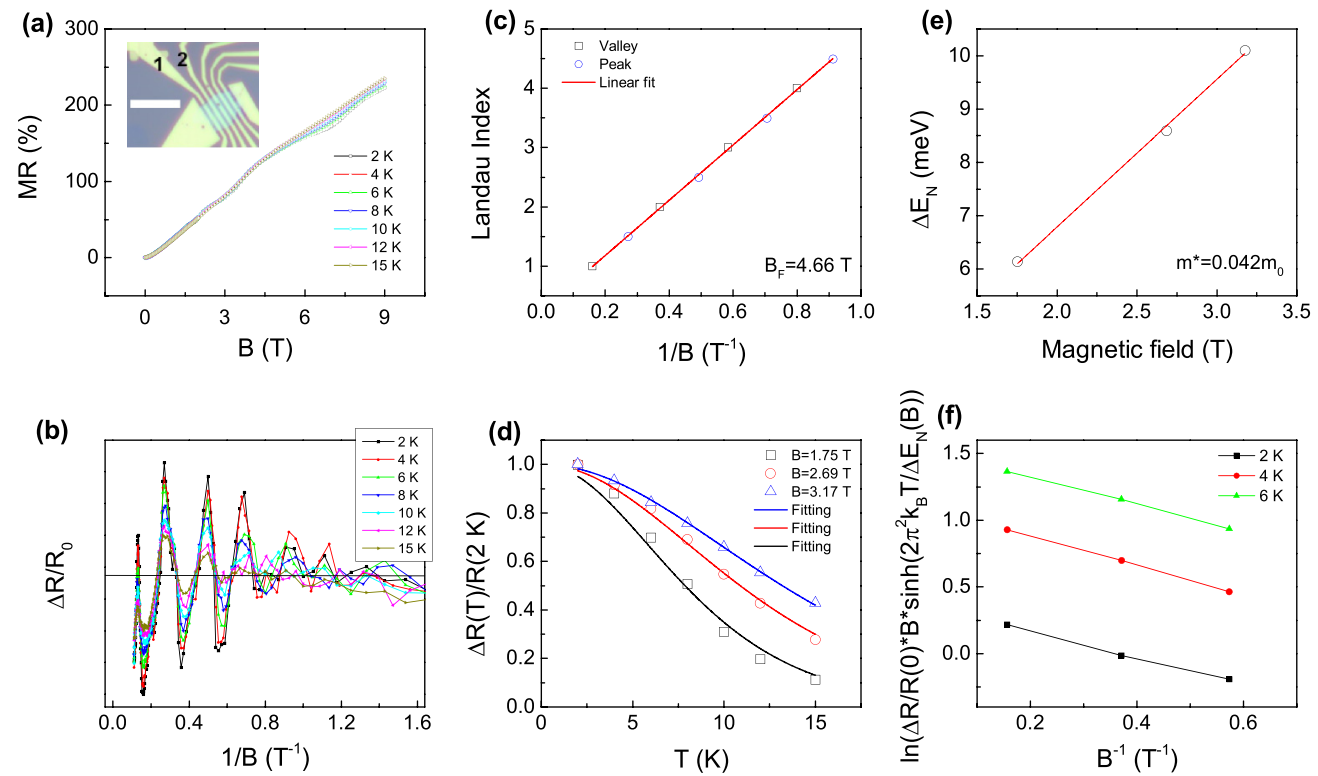

FIG. 2. (a) Field dependence of MR of a microscaled multilayered graphene sheet separated from the graphene foams measured at low temperatures. The inset is the optical image of the sample with micropatterned electrical contacts, in which the scale bar (white) is $5 \mu \mathrm{m}$. MR was measured between electrodes 1 and 2. (b) Plot of $\Delta R / R_{0}$ (linear background subtracted) as a function of $B^{-1}$ at different temperatures. (c) Landau level fan diagram for oscillations in $\Delta R$. An oscillation frequency of $B_{\mathrm{F}} \sim 4.66 \mathrm{~T}$ was obtained using the slope of the linear fit. (d) Temperature dependence of the $\mathrm{SdH}$ oscillation amplitude $\Delta R / R_{0}$ at different magnetic fields. The solid lines are the fits to the Lifshitz-Kosevich formula. (e) Magnetic field dependent Landau energy difference $\Delta E_{N}(\mathrm{~B})$. The effective mass is obtained from the slope, $m *=0.042 m_{0}$. (f) Dingle plots of $\ln \left[\frac{\Delta R}{R(0)} \mathrm{B} \sinh \left(\frac{2 \pi^{2} k_{\mathrm{B}} T}{\Delta E_{N}}\right)\right]$ vs $B^{-1}$ at different temperatures.

The Fermi velocity is obtained as $v_{F}=\hbar k_{F} / m^{*}=1.055 \times$ $10^{-34} \times 1.37 \times 10^{8} / 0.381 \times 10^{-31} \approx 3.35 \times 10^{5} \mathrm{~ms}^{-1}$.

Now, we compare the Fermi energy with the Landau level separation and examine whether the extreme quantum condition is satisfied in this sample. If we take the largest magnetic field $(B=9 \mathrm{~T})$, the separation of Landau levels is $\hbar \omega_{C}=\frac{\hbar e \mathrm{~B}}{m *}=\frac{\hbar e \times 9 \mathrm{~T}}{0.042 m_{0}}=25.90 \mathrm{meV}$, which is still smaller than the Fermi energy, $E_{F}=\hbar k_{F} v_{F} \approx 26.83 \mathrm{meV}$. It is clear that the requirement of extreme quantum condition, $\hbar \omega_{c}>$ $E_{F}$, is not satisfied here. Therefore, the quantum model can be excluded as a possible origin for the strong LMR observed in the graphene foam.

More information can be obtained about the electrical transport in our sample from the above results. The Dingle temperature, $T_{\mathrm{D}}=1.7 \mathrm{~K}$, was extracted from a semilogarithmic plot of $\frac{\Delta R}{R(0)} B \sinh \left(\frac{2 \pi^{2} k_{\mathrm{B}} T}{\Delta E_{N}}\right)$ vs $B^{-1}$, as shown in Fig. 2(f). Using the $T_{\mathrm{D}}$ value, we obtained the quantum lifetime $\tau_{q}=$ $7.16 \times 10^{-13} \mathrm{~s}$ and quantum mobility $\mu_{q}=e v_{F} \tau_{q} / \hbar k_{F} \approx$ $26,500 \mathrm{~cm}^{2} \mathrm{~V}^{-1} \mathrm{~s}^{-1}$. The classical transport scattering time $\left(\tau_{t}\right)$ is defined as the average time between the two scattering events in the diffusion transport, whereas quantum scattering time $\tau_{q}$ is a measure of the time of a carrier staying in a momentum eigenstate, with Landau level broadening. Therefore, the mean free path of the carrier transport should be calculated using $\tau_{t}$. However, the transport scattering time $\tau_{t}$ is connected to quantum scattering time $\tau_{q}$ by $\tau_{t} \cong$ $\left(E_{F} / \hbar\right)^{1 / 2} \tau_{q}^{3 / 2}[24,39,40]$. The calculated transport scattering time in our microscaled graphene sheet is $3.15 \times 10^{-12} \mathrm{~s}$ with a ratio of $\tau_{t} / \tau_{q} \sim 4.4$. Therefore, the mean free path in this sample is $l=v_{F} \tau_{t} \approx 1047 \mathrm{~nm}$.
Since the quantum model is not applicable to interpret the strong LMR in this sample, we then turn to the classical PL model and examine whether the strong LMR can be accounted for within this model. Spatial mobility fluctuations that originate from disorder and inhomogeneity in the materials are the key elements that result in LMR within the classical PL model. If the mobility fluctuation is smaller than the average mobility, $\Delta \mu /\langle\mu\rangle<1$ (first case), the classical PL model predicts that the crossover field, $H_{\mathrm{C}}$ (the critical magnetic field at which the MR becomes linear), should be linearly proportional to $\langle\mu\rangle^{-1}$ and that MR is proportional to the average mobility, $\mathrm{MR} \propto\langle\mu\rangle$ [18]. The crossover fields were then obtained from the first-order derivative of the MR data with respect to the magnetic field; the inset in Fig. 3(a) shows an example of how to determine $H_{\mathrm{C}}$ [41]. The average
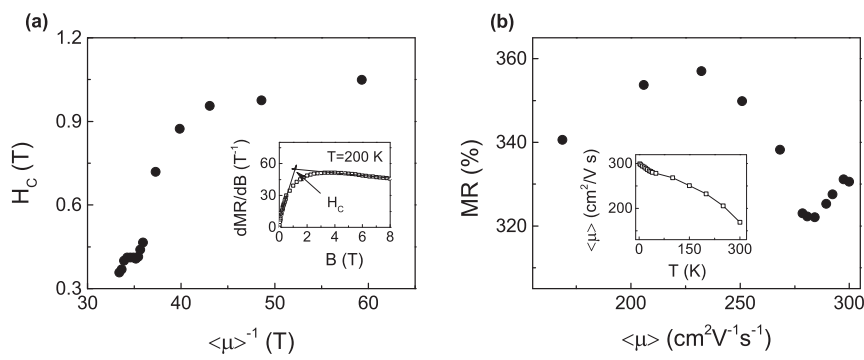

FIG. 3. (a) Plot of crossover field, $H_{\mathrm{C}}$, versus average mobility, $\langle\mu\rangle^{-1}$. The inset shows an example of how $H_{\mathrm{C}}$ is determined. (b) The dependence of average mobility, $\langle\mu\rangle$, on MR at different temperatures. The inset shows the temperature-dependent average classical mobility. 

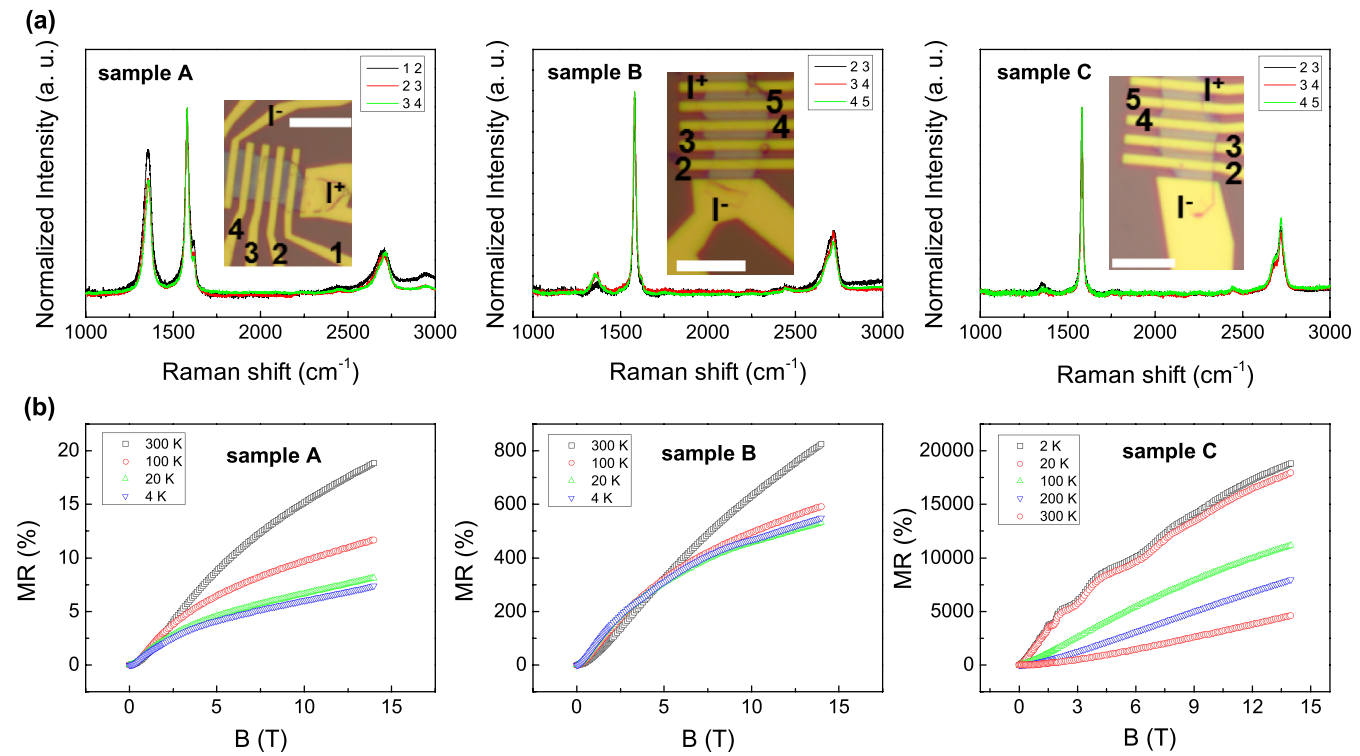

FIG. 4. (a) Micro-Raman spectra of three graphene sheets from graphene foams. The insets are the optical images of microscaled samples after nanofabrication. The white scale bar in optical images is $5 \mu \mathrm{m}$. (b) Magnetic field dependence of MR of three samples (between electrodes 2 and 3) obtained at various temperatures.

mobility of millimeter-sized graphene foam (large sample) was calculated using $\mu=R_{H} / \rho_{x x}$, where $R_{H}$ is the Hall coefficient obtained from the data shown in the inset of Fig. 1(c). The inset of Fig. 3(b) displays the temperature dependence of the calculated average mobility. The dependence of $H_{C}$ on the average mobility, $\langle\mu\rangle^{-1}$, and the dependence of MR on $\langle\mu\rangle^{-1}$ are shown in Figs. 3(a) and 3(b), respectively. Neither of these relationships shows linear dependence for graphene foam. Therefore, our results do not meet the requirements of the first case of the classical PL model, $\Delta \mu /\langle\mu\rangle<1$.

The second case of the classical PL model requires that $\Delta \mu /\langle\mu\rangle>1$, such that LMR is predicted to be proportional to a large spatial mobility fluctuation, $\Delta \mu$. To examine the spatial mobility fluctuation of graphene from different regions, the MR effects of more microscaled graphene sheets were investigated. To obtain information about the defects in the graphene sheets, we collected micro-Raman spectra from all the samples after patterning of the electrodes, as shown in Fig. 4(a). As is well known, the strength of the D peak (at $1350 \mathrm{~cm}^{-1}$ ) in the Raman spectrum reflects the density of defects in the graphene. As seen in Fig. 4(a), much more disorder or more defects are present in sample A than in sample $B$, and the least defects exist in sample C. To explore how the defects affect the electrical transport properties in these graphene sheets, we measured the MR at various temperatures [Fig. 4(b)]. As shown in Fig. 4(b), the maximum MR in sample A (the dirtiest sample) is less than $20 \%$, which is much smaller than $\sim 800 \%$ in sample B. Sample C (the cleanest sample) shows a giant MR of $\sim 20,000 \%$. Interestingly, the $\mathrm{SdH}$ oscillations are discernible from the giant and positive MR data obtained from samples B and C directly without the data analysis as we did for the data in Fig. 1(d). By fitting the low-field $(B<0.5 \mathrm{~T}) \mathrm{MR}$ data in Fig. $4(\mathrm{~b})$ to $\mathrm{MR}=(\mu \mathrm{B})^{2}$, we obtained the average mobility of the microscaled graphene sheets. The average mobility values of samples $\mathrm{B}$ and $\mathrm{C}$ between electrodes 2-3, electrodes 3-4, and electrodes 4-5 are, respectively, 5250, 8240, and $2370 \mathrm{~cm}^{2} \mathrm{~V}^{-1} \mathrm{~s}^{-1}$ (sample B) and 64,200, 32,700, and 54,200 $\mathrm{cm}^{2} \mathrm{~V}^{-1} \mathrm{~s}^{-1}$ (sample C). On the contrary, the fitted average mobility values of sample A are significantly smaller, i.e., 1520, 1370, and $1850 \mathrm{~cm}^{2} \mathrm{~V}^{-1} \mathrm{~s}^{-1}$ for the region between electrodes $1-2$, electrodes 2-3, and electrodes 3-4, respectively. Evidently, the reduced mobility can be attributed to the high density/strength of defects in sample A [Fig. 4(a)], which largely suppressed MR in comparison with that observed in samples B and C. This experiment allows us to measure the local mobility at random locations, which is due to the unique characteristics of the graphene foam: (a) The foam is formed by microsized graphene sheets; (b) the foam can be broken into individual microsized graphene sheets; and (c) the microsized sheets are large enough for electrical measurements.

It is clear that the spatial mobility fluctuation is very strong and large across a graphene foam with a size of a few millimeters, and this results in the giant, positive LMR in the 3D graphene foams based on the PL model. Furthermore, the observed SdH oscillations in the millimetersized graphene foam in Fig. 2 demand that $\omega_{c} \tau \gg 1$ or $\mu B \gg 1$, where $\omega_{c}$ is the cyclotron frequency, and $\tau$ is the quantum lifetime. As shown in Fig. 3(b), the measured average mobility of millimeter-sized graphene foam was relatively small, $\langle\mu\rangle=150-300 \mathrm{~cm}^{2} \mathrm{~V}^{-1} \mathrm{~s}^{-1}$. Therefore, the average mobility in the large graphene foam sample will not lead to the $\mathrm{SdH}$ oscillations. The observed $\mathrm{SdH}$ oscillations must have originated from the micron-sized, well-crystallized graphene sheets with large enough mobility, such as samples B and C (Fig. 4), and particularly the sample C type of micron sheets. The size of the perfect/clean graphene sheets may be $8-10 \mu \mathrm{m}$, as determined by the $\mathrm{Ni}$ grain size of the Ni skeleton as shown in Fig. 1(b). Next, we look at the mobility fluctuation in the large graphene foam sample. Taking the largest average mobility as high as $64,200 \mathrm{~cm}^{2} \mathrm{~V}^{-1} \mathrm{~s}^{-1}$ observed in a microscaled graphene sheet (sample $\mathrm{C}$ ) as a local 
mobility and the average mobility measured in the millimeter foam, the largest mobility fluctuation should be estimated as $\frac{\Delta \mu}{\langle\mu\rangle} \sim \frac{64200-300}{300} \approx 213 \gg 1$, which satisfies the requirement of the second case of the classical PL model. Even when we take a mobility of $1370 \mathrm{~cm}^{2} \mathrm{~V}^{-1} \mathrm{~s}^{-1}$, obtained from sample A, the mobility fluctuation $\frac{\Delta \mu}{\langle\mu\rangle} \sim \frac{1370-300}{300}=3.56>1$. So the precondition, $\Delta \mu /\langle\mu\rangle>1$, for the second case of the PL model can be easily satisfied in our graphene foam. Samples A, $\mathrm{B}$, and $\mathrm{C}$ were chosen because of their high crystalline quality based on the optical image before patterning. Nevertheless, within these three high-quality samples, the mobility can vary from 1370 to $64,200 \mathrm{~cm}^{2} \mathrm{~V}^{-1} \mathrm{~s}^{-1}$, i.e., more than 45 times difference. Even within a single clean microsheet (sample A, B, or C in Fig. 4), the mobility fluctuation between different electrodes can be quite large. For a big, millimetersized graphene foam, there must be significant numbers of microsheets with more defects, particularly in the area of the grain boundaries, which have much lower mobility. These wrinkles, ripples, and particularly the very defective grain boundaries lead to very strong mobility fluctuation and a relatively small average mobility, $<300 \mathrm{~cm}^{2} \mathrm{~V}^{-1} \mathrm{~s}^{-1}$. Based on the PL model, the very strong mobility fluctuation and a relatively small average mobility will lead to observation of a giant, positive LMR, which was clearly observed in the graphene foam in this study.

\section{CONCLUSIONS}

In summary, LMR was observed in 3D networked graphene foam. A LMR this large is nearly temperature independent between $2 \mathrm{~K}$ and $300 \mathrm{~K}$ in macroscopical graphene foams. Careful analysis of the MR data reveals clear $\mathrm{SdH}$ oscillations at low temperatures that attenuate with increasing temperatures. $\mathrm{SdH}$ oscillations are ascribed to the intrinsic characteristics of graphene, such as large mobility and a small electron mass within individual graphene grains of several microns in size. Observations of $\mathrm{SdH}$ oscillations exclude the possibility that the large LMR originated from the extreme quantum conditions proposed by Abrikosov. We successfully extracted the mobility fluctuation by measuring local mobility on the microsized graphene sheets. Ultimately, the coexistence of LMR with SdH oscillations has been interpreted within the classical PL model for multilayered graphene foam, where huge spatial mobility fluctuation related to large amounts of defects, such as at the grain boundaries and ripples and wrinkles, dominates electrical transport. This study serves as a strong confirmation of the PL model. This study has clarified the physics behind the large LMR in graphene materials and is important for practical applications in magnetic sensors that work in broad temperature and magnetic field ranges.

\section{ACKNOWLEDGMENTS}

P.L. was supported by a Saudi Basic Industries Corporation (SABIC) Postdoctoral Fellowship Award in the Kingdom of Saudi Arabia. H.-M.C. and W.R. acknowledge the financial support by the National Natural Science Foundation of China (Grant No. 51221264), the Ministry of Science and Technology of China (Grant No. 2012AA030303), and the Chinese Academy of Sciences (Grants No. KGZD-EW-303-1 and No. KGZD-EW-T06). We are grateful to Jun Li and Zhiyong Zhu for helpful discussions. This work was mainly supported by the King Abdullah University of Science and Technology.
[1] R. Xu, A. Husmann, T. F. Rosenbaum, M. L. Saboungi, J. E. Enderby, and P. B. Littlewood, Nature 390, 57 (1997).

[2] M. Lee, T. F. Rosenbaum, M. L. Saboungi, and H. S. Schnyders, Phys. Rev. Lett. 88, 066602 (2002).

[3] J. S. Hu and T. F. Rosenbaum, Nat. Mater. 7, 697 (2008).

[4] X. L. Wang, Y. Du, S. X. Dou, and C. Zhang, Phys. Rev. Lett. 108, 266806 (2012).

[5] Z. H. Wang, L. Yang, X. J. Li, X. T. Zhao, H. L. Wang, Z. D. Zhang, and X. P. A. Gao, Nano Lett. 14, 6510 (2014).

[6] M. N. Ali, J. Xiong, S. Flynn, J. Tao, Q. D. Gibson, L. M. Schoop, T. Liang, N. Haldolaarachchige, M. Hirschberger, N. P. Ong, and R. J. Cava, Nature 514, 205 (2014).

[7] T. Liang, Q. Gibson, M. N. Ali, M. H. Liu, R. J. Cava, and N. P. Ong, Nat. Mater. 14, 280 (2015).

[8] A. Narayanan, M. D. Watson, S. F. Blake, N. Bruyant, L. Drigo, Y. L. Chen, D. Prabhakaran, B. Yan, C. Felser, T. Kong, P. C. Canfield, and A. I. Coldea, Phys. Rev. Lett. 114, 117201 (2015).

[9] M. Novak, S. Sasaki, K. Segawa, and Y. Ando, Phys. Rev. B 91, 041203 (2015).

[10] A. L. Friedman, J. L. Tedesco, P. M. Campbell, J. C. Culbertson, E. Aifer, F. K. Perkins, R. L. Myers-Ward, J. K. Hite, C. R. Eddy, Jr., G. G. Jernigan, and D. K. Gaskill, Nano Lett. 10, 3962 (2010).
[11] R. S. Singh, X. Wang, W. Chen, Ariando, and A. T. S. Wee, Appl. Phys. Lett. 101, 183105 (2012).

[12] Z. M. Liao, H. C. Wu, S. Kumar, G. S. Duesberg, Y. B. Zhou, G. L. W. Cross, I. V. Shvets, and D. P. Yu, Adv. Mater. 24, 1862 (2012).

[13] R. Mahendiran, A. K. Raychaudhuri, A. Chainani, and D. D. Sarma, Rev. Sci. Instrum. 66, 3071 (1995).

[14] S. A. Solin, T. Thio, D. R. Hines, and J. J. Heremans, Science 289, 1530 (2000).

[15] A. Husmann, J. B. Betts, G. S. Boebinger, A. Migliori, T. F. Rosenbaum, and M. L. Saboungi, Nature 417, 421 (2002).

[16] P. P. Freitas, R. Ferreira, S. Cardoso, and F. Cardoso, J. Phys. Condens. Mat. 19, 165221 (2007).

[17] C. Xia, Q. Jiang, C. Zhao, P. M. Beaujuge, and H. N. Alshareef, Nano Energy 24, 78 (2016).

[18] M. M. Parish and P. B. Littlewood, Nature 426, 162 (2003).

[19] M. M. Parish and P. B. Littlewood, Phys. Rev. B 72, 094417 (2005).

[20] A. A. Abrikosov, Phys. Rev. B 58, 2788 (1998).

[21] A. A. Abrikosov, Europhys. Lett. 49, 789 (2000).

[22] S. O. Demokritov, A. A. Serga, V. E. Demidov, B. Hillebrands, M. P. Kostylev, and B. A. Kalinikos, Nature 426, 159 (2003). 
[23] Z. M. Liao, Y. B. Zhou, H. C. Wu, B. H. Han, and D. P. Yu, Europhys. Lett. 94, 57004 (2011).

[24] X. Hong, K. Zou, and J. Zhu, Phys. Rev. B 80, 241415 (2009).

[25] S. Dusari, J. Barzola-Quiquia, P. Esquinazi, and N. Garcia, Phys. Rev. B 83, 125402 (2011).

[26] H. K. Pal and D. L. Maslov, Phys. Rev. B 88, 035403 (2013).

[27] Z. P. Chen, W. C. Ren, L. B. Gao, B. L. Liu, S. F. Pei, and H. M. Cheng, Nat. Mater. 10, 424 (2011).

[28] See Supplemental Material at http://link.aps.org/supplemental/ 10.1103/PhysRevB.94.045402 for nanofabrication process of electrodes for graphene sheet.

[29] S. J. Chae, F. Gunes, K. K. Kim, E. S. Kim, G. H. Han, S. M. Kim, H. J. Shin, S. M. Yoon, J. Y. Choi, M. H. Park, C. W. Yang, D. Pribat, and Y. H. Lee, Adv. Mater. 21, 2328 (2009).

[30] X. S. Wu, X. B. Li, Z. M. Song, C. Berger, and W. A. de Heer, Phys. Rev. Lett. 98, 136801 (2007).

[31] D. X. Qu, Y. S. Hor, J. Xiong, R. J. Cava, and N. P. Ong, Science 329, 821 (2010).

[32] Z. Ren, A. A. Taskin, S. Sasaki, K. Segawa, and Y. Ando, Phys. Rev. B 82, 241306 (2010).
[33] J. G. Analytis, J. H. Chu, Y. L. Chen, F. Corredor, R. D. McDonald, Z. X. Shen, and I. R. Fisher, Phys. Rev. B 81, 205407 (2010).

[34] K. Eto, Z. Ren, A. A. Taskin, K. Segawa, and Y. Ando, Phys. Rev. B 81, 195309 (2010).

[35] See Supplemental Material at http://link.aps.org/supplemental/ 10.1103/PhysRevB.94.045402 for detailed anisotropic magnetoresistance analysis for graphene foams.

[36] See Supplemental Material at http://link.aps.org/supplemental/ 10.1103/PhysRevB.94.045402 for magnetoresistance of nickel skeleton and magnetization of graphene foam.

[37] Y. B. Zhang, Y. W. Tan, H. L. Stormer, and P. Kim, Nature 438, 201 (2005).

[38] C. Berger, Z. M. Song, X. B. Li, X. S. Wu, N. Brown, C. Naud, D. Mayou, T. B. Li, J. Hass, A. N. Marchenkov, E. H. Conrad, P. N. First, and W. A. de Heer, Science 312, 1191 (2006).

[39] R. D. Brown, Phys. Rev. B 2, 928 (1970).

[40] T. A. Komissarova, V. N. Jmerik, S. V. Ivanov, O. Drachenko, X. Wang, and A. Yoshikawa, Phys. Rev. B 84, 035205 (2011).

[41] W. H. Wang, Y. Du, G. Z. Xu, X. M. Zhang, E. K. Liu, Z. Y. Liu, Y. G. Shi, J. L. Chen, G. H. Wu, and X. X. Zhang, Sci. Rep. 3, 2181 (2013). 